\title{
Parasympathetic, Sympathetic, and Sensory Interactions in the Iris: Nerve Growth Factor Regulates Cholinergic Ciliary Ganglion Innervation In Vivo'
}

\author{
JOHN A. KESSLER \\ Departments of Neurology and Neuroscience, Albert Einstein College of Medicine, Bronx, New York 10461
}

\begin{abstract}
Interactions between peptidergic sensory nerves, noradrenergic sympathetic nerves, and cholinergic parasympathetic fibers were examined in the rat iris. The putative peptide neurotransmitter, substance $P$ (SP), was used as an index of the trigeminal sensory innervation, tyrosine hydroxylase (TH) activity served to monitor the sympathetic fibers, and choline acetyltransferase (CAT) activity was used as an index of the parasympathetic innervation. Destruction of the sympathetic innervation by neonatal administration of 6-hydroxydopamine resulted in increased SP development and a smaller increase in CAT activity in the iris. Moreover, trigeminal ablation resulted in an increase in both TH and CAT activities. Finally, ciliary ganglionectomy resulted in increased SP and a smaller increase in TH activity in the iris. Administration of nerve growth factor (NGF) into the anterior chamber substantially increased both SP and TH activity in the iris and also increased CAT activity to a lesser extent. Moreover, administration of anti-NGF into the anterior chamber prevented both the sympathectomy-induced increases in SP and CAT, and the increases in TH and CAT activities after trigeminal ablation, suggesting that NGF mediated these increases. These observations suggest that the sympathetic, sensory, and parasympathetic innervations of the iris interact by altering availability of NGF elaborated by the iris. Regulation of iris CAT activity was examined in greater detail. Injection of the cholinergic toxin, AF64A, into the anterior chamber concurrently with ablation of the sympathetic and sensory innervations paradoxically increased CAT activity, whereas AF64A alone decreased CAT activity. Moreover, injection of the anterior chamber with NGF after AF64A administration also increased CAT activity more than NGF treatment alone, suggesting that damaged or regenerating ciliary fibers are more responsive to NGF than the intact innervation. These observations suggest that the balance between the sympathetic, sensory, and parasympathetic innervations of the iris may be regulated by availability of NGF elaborated by the iris.
\end{abstract}

Neuronal interactions with target tissues are essential for both the development of immature neurons (Hamburger, 1934; Prestige,

Received November 19, 1984; Revised February 7, 1985; Accepted March 4, 1985

${ }^{1}$ I would like to thank Ms. Kathryn Sweeney for her outstanding technical assistance, and Ms. Antoinette Barnecott for help in preparing the manuscript. This work was supported by National Institutes of Health Grants 20013 and 20778, and by a grant from the March of Dimes.
1967; Hendry and Iversen, 1973; Landmesser and Pilar, 1974; Kessler and Black, 1980a) and for the maintenance of adult phenotypic traits (Olson and Malmfors, 1970; Hendry and Thoenen, 1974). At least some of the interactions between neurons and their targets appear to be mediated by chemical factors capable of supporting survival and development of responsive neurons. For example, recent studies suggest that some innervating neurons and their targets exert reciprocal effects through the mediation of nerve growth factor (NGF). Targets regulate sympathetic (Hendry and Thoenen, 1974) and peptidergic sensory (Kessler and Black, 1980a; Otten et al., 1980) neurons via this trophic protein and, conversely, sympathetic and sensory innervations appear to influence target elaboration of NGF (Ebendal et al., 1980). These target regulatory processes may be extremely complex, involving interactions among multiple co-innervating neuronal populations and the target itself. In the iris, for example, we recently found that peptidergic sensory nerves are modulated by co-innervating sympathetic fibers (Kessler et al., 1983b); extirpation of the innervating sympathetic superior cervical ganglion (SCG) increased levels of the putative peptide neurotransmitter, substance P (SP) (Kessler et al., 1983b), which is localized in the iris to trigeminal sensory fibers (Cuello et al., 1978; Butler et al., 1980; Tervo et al., 1982; Miller et al., 1981; Kessler et al., 1983a). Injection of NGF into the anterior chamber of the eye increased iris $\mathrm{SP}$, reproducing the effects of sympathectomy. Conversely, injection of anti-NGF into the anterior chamber prevented the increase in peptide after sympathetic ablation, suggesting that co-innervating sympathetic nerves interacted indirectly with sensory fibers by influencing elaboration of NGF by their mutual target.

The SCG and the trigeminal ganglion (TG) are not the only major sources of innervation of the iris, which also receives cholinergic fibers from the parasympathetic ciliary ganglion (CG). The present communication examines interactions in the iris between these three sources of innervation. SP is used to monitor the trigeminal innervation, since the peptide is restricted to these fibers in the iris Tyrosine hydroxylase ( $\mathrm{TH}$ ), the rate-limiting enzyme in norepinephrine synthesis (Levitt et al., 1965), is used as an index of the sympathetic noradrenergic innervation, whereas choline acetyltransferase (CAT) serves as an index of the cholinergic, CG fibers. Our observations suggest that CG neurons, as well as TG and SCG neurons, are responsive to NGF in vivo, and that the availability of NGF elaborated by the iris regulates maintenance of the sympathetic, sensory, and parasympathetic innervations.

\section{Materials and Methods}

\section{Experimental animals}

Sprague-Dawley rats weighing 150 to $225 \mathrm{gm}$ (CAMM Research Lab Animals) were housed in clear plastic and wire cages and were exposed to 550 to 800 lux of cool white fluorescent illumination from 5:00 A.M. to 6:30 P.M. daily. Ralston Purina Lab Chow and water were offered ad libitum. 


\section{Biochemical procedures}

TH activity was assayed as previously described utilizing tetrahydrobiopterin as cofactor (Kessler and Black, 1979). CAT was assayed employing minor modifications of published methods (Fonnum, 1969). SP was measured by radioimmunoassay as described previously (Kessler and Black, 1980a)

\section{Preparation of NGF and anti-NGF}

$\beta$-NGF was prepared from adult male mouse salivary glands by the method of Mobley et al. (1976). Anti-NGF was generated as described previously (Kessler and Black, 1980b) by immunizing New Zealand White rabbits with mouse $\beta$-NGF. Biologic activity of the anti-NGF was approximately 11,000 units/ml as measured by the method of Fenton (1970). Control serum for the anti-NGF experiments was preimmune serum from the rabbit which generated the anti-NGF.

\section{Surgical procedures}

All rats were anesthetized with halothane ( $3 \%$ in $100 \%$ oxygen at 2.5 liters/min). Trigeminal ablation consisted of craniotomy, excision of a portion of the frontal lobe to expose the trigeminal nerve, and division of the first division near the exit from the TG. Care was taken to avoid sectioning the nerve near the entry into the orbit, since the sympathetic innervation travels with the nerve at this point. Sham operation consisted of exposure of the first division of the trigeminal nerve. Ciliary ganglionectomy, which was exceedingly difficult, was performed by anesthetizing the rat and entering the orbit inferolaterally. Use of a dissecting microscope $(x 20)$ was necessary for this procedure. The CG, which was localized between the optic nerve and the lateral rectus, was removed by carefully squeezing it with sharpened microforceps. The nasociliary nerve was always identified and carefully avoided; if it could not be identified or if it was fused with the CG, the animal was discarded. Frequently the ciliary ganglion could not be visualized, in which case the animal was discarded. Animals were also discarded if excess bleeding occurred. After the animals awakened the eye was examined; animals were discarded if there was not dilation of the pupil, or if the corneal reflex was impaired. After the procedure was learned, successful surgery was performed on approximately one animal out of seven. Sham operation consisted of exposure of the CG.

\section{Drug treatments}

6-Hydroxydopamine. Neonates were injected with 6-hydroxydopamine (50 mg/kg, s.c., in saline containing $0.4 \mathrm{mg}$ of ascorbic acid/ml) or buffer on days 1, 3, 5, and 7 of life to destroy sympathetic neurons (Angeletti and Levi-Montalcini, 1970; Angeletti, 1971; Jaim-Etcheverry and Zieher, 1971; Clark et al., 1972; Finch et al., 1973). In previous studies we found loss of greater than $90 \%$ of $\mathrm{TH}$ activity from the SCG with this regimen (Kessler and Black, 1982).

Capsaicin. Neonates were treated with capsaicin $(50 \mathrm{mg} / \mathrm{kg}$, s.c.) on days $1,2,3,4$, and 5 of life to destroy peptide-containing trigeminal neurons. (For review, see Nagy, 1982; see also Jancso et al., 1977; Nagy et al., 1981; Gamse et al., 1980.)

Anterior chamber injections. Injections of drugs (AF64A, NGF, or antiNGF) or vehicle into the anterior chamber of the eye were performed by anesthetizing the rat with halothane and slowly injecting $10 \mu \mathrm{l}$ using a 30 gauge needle and a Hamilton syringe. Care was taken to avoid increasing intraocular pressure rapidly. Injections were performed as far laterally as possible; ambient light conditions were kept dark to maximize iris dilation. AF64A was prepared as previously described (Mantione et al., 1981), and drug $(10 \mathrm{nmol})$ or buffer was injected daily for 2 days. NGF $(4 \mu \mathrm{g})$ or antiNGF (100 B.U.) was injected daily for 7 or 10 days depending on the experiment. Some animals (approximately one out of every seven or eight) developed corneal cloudiness and were discarded.

\section{Statistics}

Data were analyzed by the Student's $t$ test or by analysis of variance.

\section{Drugs}

Naphthylvinylpyridine was generously supplied by Dr. Robert Hamill. AF64A was purchased from Research Biochemicals, Inc. (Wayland, MA). All other drugs were purchased from Sigma Chemical Co. (St. Louis, MO).

\section{Results}

Localization of SP, TH, and CAT in the iris. To determine whether $\mathrm{SP}, \mathrm{TH}$, and CAT activities could be used as indices of the TG,
SCG, and CG innervations, respectively, iris content of these traits was examined after ablation of the appropriate structure (Table I). Trigeminal axotomy resulted in a loss of $96 \%$ of iris SP. Moreover, superior cervical ganglionectomy resulted in a $97 \%$ loss of iris $\mathrm{TH}$ activity, whereas ciliary ganglionectomy resulted in greater than $97 \%$ decrease in iris CAT activity. Thus, the anatomic restriction of these traits to separate innervations allowed their use as indices of the sensory, sympathetic, and parasympathetic innervations.

Iris CAT activity was assayed by a modified technique (Fonnum, 1969) which is not necessarily specific for CAT; carnitine acetylase, for example, may cross-react in the assay (White and Wu, 1973). Iris homogenates were therefore assaycd both in the absence and in the presence of the specific inhibitor of CAT, naphthylvinylpyridine (White and $\mathrm{Wu}, 1973$ ). The inhibitor blocked $97.8 \%$ of enzyme activity in the iris, indicating that measured activity represented authentic CAT (Table II). Moreover, naphthylvinylpyridine inhibited $98.4 \%$ of enzyme activity after NGF treatment and $98.3 \%$ of activity after combined sympathetic and trigeminal ablations, indicating that CAT activity measured after these procedures represented authentic enzyme (Table II).

Effects of 6-hydroxydopamine. To examine influences of the sympathetic innervation of the iris on the sensory and parasympathetic innervations, neonates were injected with 6-hydroxydopamine to destroy sympathetic nerves (Angeletti and Levi-Montalcini, 1970; Angeletti, 1971; Jaim-Etcheverry and Zieher, 1971; Clark et al., 1972; Finch et al., 1973). Animals were allowed to mature, and the irides were examined for content of SP and CAT (Fig. 1). Irides in vehicletreated controls contained $76 \mathrm{pg}$ of SP/iris. Administration of 6 hydroxydopamine more than doubled peptide content to $159 \mathrm{pg} /$ iris as previously reported (Kessler et al., 1983b). By contrast, 6-hydroxydopamine treatment elevated CAT activity by $16 \%$. However, concurrent treatment with both 6-hydroxydopamine and capsaicin, a drug which destroys SP-containing sensory nerves in neonates (for review, see Nagy, 1982), significantly elevated CAT activity by $39 \%$, suggesting that sympathetic and sensory fibers also interact with the cholinergic, parasympathetic innervation.

Effects of trigeminal ganglionectomy. To examine influences of the sensory innervation, unilateral trigeminal axotomy or sham op-

\section{TABLE 1}

Anatomic localization of neurotransmitter traits in the iris

Irides were examined for content of SP (mean picograms per iris \pm SEM), $\mathrm{TH}$ activity (mean picomole product per iris per hour \pm SEM), and CAT activity (mean nanomole product per iris per hour \pm SEM). $N=8$ except for ciliary ganglionectomy, where $N=4$.

\begin{tabular}{lrcc}
\hline & SP & TH & CAT \\
\hline Control & $78 \pm 6.4$ & $44 \pm 3.1$ & $79 \pm 5.3$ \\
$\begin{array}{l}\text { Trigeminal axotomy } \\
\begin{array}{l}\text { Superior cervical ganglio- } \\
\quad \text { nectomy }\end{array}\end{array}$ & $3.1 \pm 0.2$ & & \\
Ciliary ganglionectomy & & $0.9 \pm 0.4$ & \\
\hline
\end{tabular}

TABLE ॥

Effects of naphthylvinylpyridine on iris CAT activity

Irides were examined for CAI activity 10 days atter unilateral combined SCG and TG ablations or after 7 days of NGF ( $4 \mu \mathrm{g}$ in $10 \mathrm{ml})$ injections into the anterior chamber. The homogenates were split, and half were assayed in the usual manner whereas half were assayed in the presence of naphthylvinylpyridine $(0.3 \mathrm{~mm})$. Values represent the mean nanomole product per iris per hour \pm SEM. $N=6$.

\begin{tabular}{|c|c|c|}
\hline & $\begin{array}{l}\text { Normal } \\
\text { Assay }\end{array}$ & $\begin{array}{c}\text { With } \\
\text { Naphthylvinylpyridine }\end{array}$ \\
\hline Control & $77 \pm 2.4$ & $1.7 \pm 0.1$ \\
\hline NGF & $101 \pm 3.2$ & $1.6 \pm 0.2$ \\
\hline TG \& SCG ablation & $111 \pm 5.3$ & $1.9 \pm 0.3$ \\
\hline
\end{tabular}




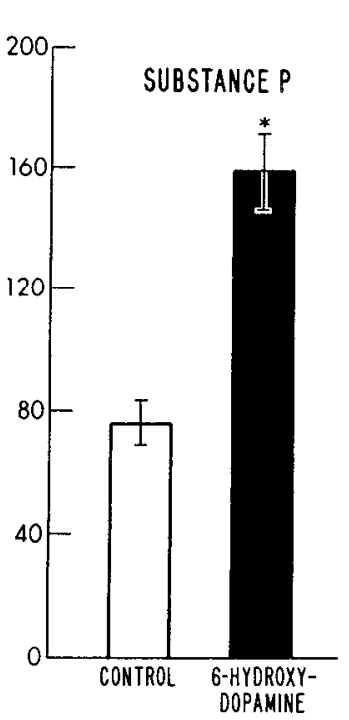

Figure 1. Effects of 6-hydroxydopamine on SP and CAT in the iris Neonates were injected with 6-hydroxydopamine $(50 \mathrm{mg} / \mathrm{kg}$, s.c., in ascorbate) or buffer on days $1,3,5$, and 7 of life. Some animals were also treated with capsaicin (50 mg/kg, s.c.) on days $1,2,3,4$, and 5 of life. Ten weeks later the irides were removed and examined for content of SP, expressed as mean picograms per iris \pm SEM, and CAT activity, expressed as mean nanomole product per iris per hour \pm SEM. $N=8$. * differs from control at $p<0.001 ; * *$, differs from control at $p<0.025$
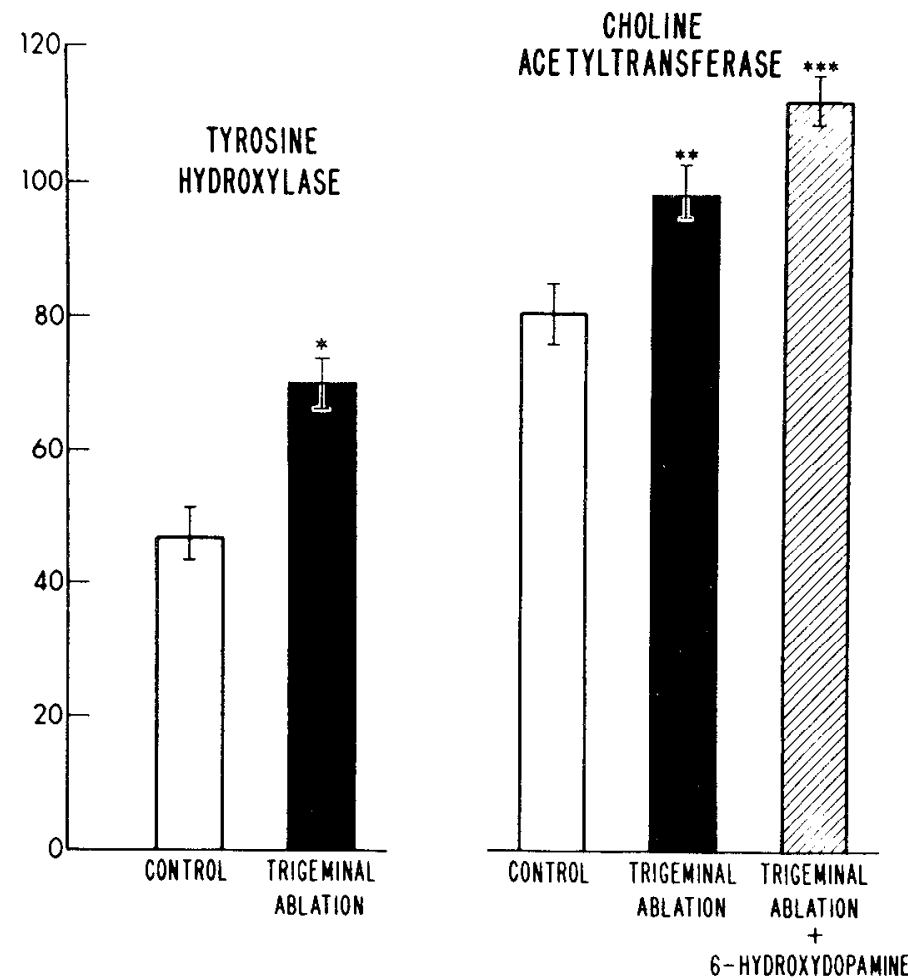

Figure 2. Effects of trigeminal axotomy on $\mathrm{TH}$ and CAT activities in the iris. Unilateral TG ablation or sham operation was performed, and 2 weeks later the irides were examined for $\mathrm{TH}$ activity, expressed as mean picomole product per iris per hour \pm SEM, and CAT activity. In some instances surgery was performed on adult animals which had been treated as neonates with 6-hydroxydopamine. $N=8$. $*$, differs from control at $\rho<0.025 ; *$, differs from control at $p<0.05 ; * * *$, differs from control at $p<0.01$. eration was performed, and 2 weeks later the irides were examined for content of TH and CAT activitics (Fig. 2). Trigeminal ablation resulted in a significant $49 \%$ increase in iris $\mathrm{TH}$ activity compared to sham operation. Moreover, trigeminal denervation also significantly elevated CAT by $25 \%$. Finally, trigeminal ablation in adult animals treated with 6-hydroxydopamine as neonates resulted in an even larger $48 \%$ increase in CAT activity.

Effects of ciliary ganglionectomy. To examine influences of the parasympathetic innervation, unilateral ciliary ganglionectomy or sham operation was performed, and 2 weeks later the irides were examined for content of SP and TH activity (Fig. 3). Ciliary ablation resulted in a significant $40 \%$ increase in $\mathrm{TH}$ activity compared to sham operation. Moreover, ciliary ganglionectomy also significantly elevated iris SP by $44 \%$, indicating that parasympathetic fibers modulate the sensory as well as sympathetic innervations of the iris.

Effects of NGF. Previously we found that administration of NGF into the anterior chamber increased iris SP, reproducing the effects of sympathectomy. Moreover, anti-NGF prevented the effects of sympathectomy on iris SP, suggesting that increased availability of NGF mediated the increase in peptide. To determine whether NGF administration also altered iris TH and CAT activities, unilateral injections of the factor or of vehicle were performed and irides were examined for content of SP, TH, and CAT (Fig. 4). There were no significant differences in SP, TH, or CAT between vehicle-treated irides and irides contralateral to the NGF injections (data not shown), excluding systemic effects of the factor. However, NGF administration resulted in an $85 \%$ increase in SP, a $59 \%$ increase in $\mathrm{TH}$, and a $29 \%$ increase in CAT activity (Fig. 4). Consequently, the parasympathetic as well as the sensory and sympathetic innervations of the iris are resporisive to NGF.

Effects of anti-NGF. Previously, we found that anti-NGF administration into the anterior chamber decreased iris SP, suggesting that endogenous NGF regulates iris peptide (Kessler et al., 1983b). Moreover, anti-NGF administration prevented the sympathectomyinduced increase in SP, suggesting that NGF mediated the sensorysympathetic interaction. To examine the effects of anti-NGF treatment on the sympathetic and parasympathetic innervations, antiserum was injected into the anterior chamber of control animals and of animals undergoing SCG or TG ablation. Administration of anti-NG $\Gamma$ to unoperated animals significantly decreased iris SP by $35 \%$ and $\mathrm{TH}$ activity by $56 \%$ (Table III). By contrast, anti-NGF administration did not significantly alter CAT activity. These obser-

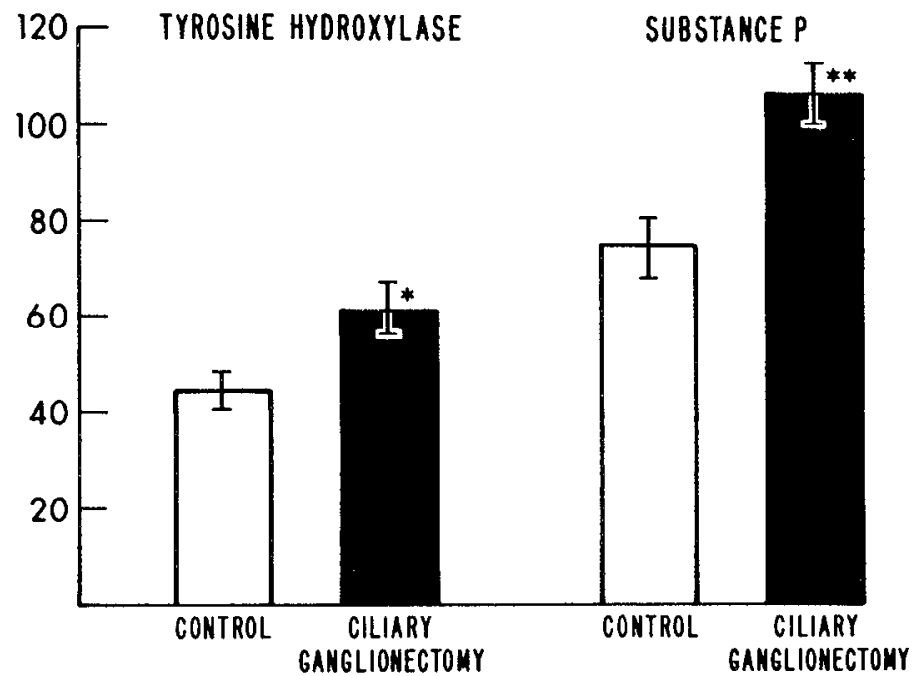

Figure 3. Effects of ciliary ganglionectomy on SP and TH activity in the iris. Unilateral ciliary ganglionectomy or sham operation was performed, and 2 weeks later the irides were examined for TH activity and SP. $N=4$.*, differs from control at $p<0.05 ; * *$, differs from control at $p<0.025$. 
vations suggest that endogenous NGF in the adult iris normally regulates the sensory and sympathetic innervations but may not regulate the parasympathetic innervation.

To determine whether NGF mediates the increases in iris $\mathrm{TH}$ and CAT after trigeminal ablation and the increases in SP and CAT after sympathectomy, rats were treated with anti-NGF after surgery. Immediately after unilateral sympathetic ganglionectomy or trigeminal axotomy, the ipsilateral chambers were injected daily with either control serum or anti-NGF. Ten days postoperatively the ipsilateral and contralateral irides were assayed. Iris TH activity in the contralateral irides was the same in all groups, as were iris peptide and CAT, excluding systemic effects of anti-NGF. Injection of control serum did not affect the expected rises in TH and CAT activities after trigeminal ablation (Table III). However, anti-NGF injection completely blocked the effects of denervation and, in fact, decreased iris TH to the same level in operated and control animals. Similarly, injection of control serum did not affect the expected increases in SP and CAT content after sympathectomy, but anti-NGF injection completely blocked the effects of sympathetic ablation (Table I). These observations suggest that the reciprocal interactions between

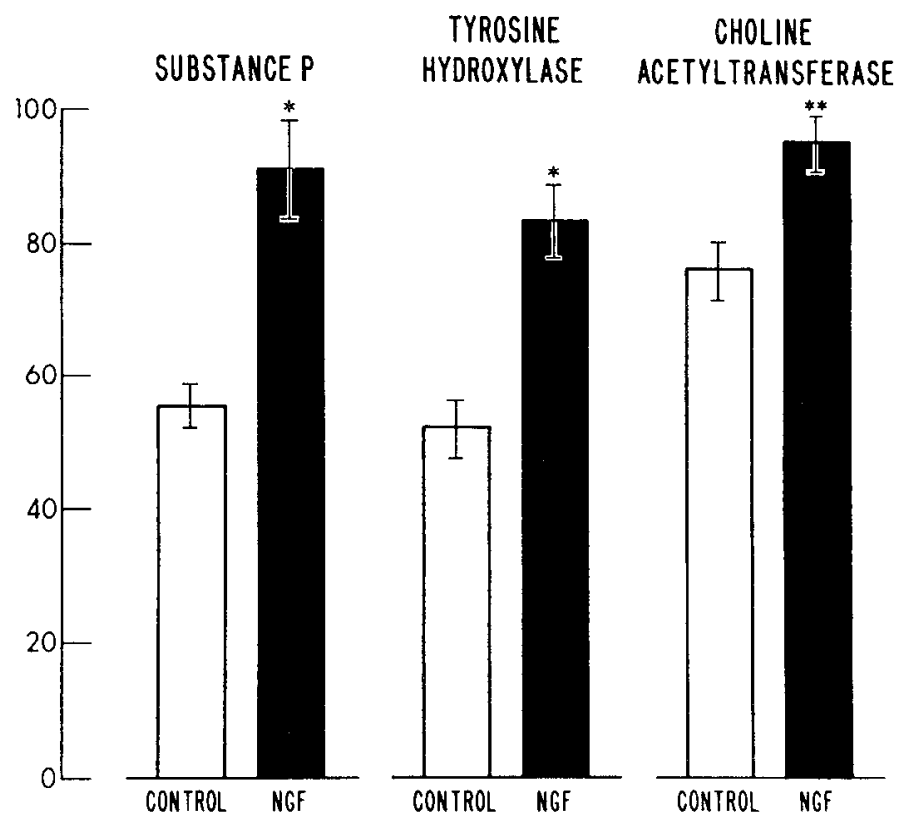

Figure 4. Effects of NGF injection into the anterior chamber of the eye. Unilateral injections of NGF $(4 \mu \mathrm{g}$ in $10 \mu$ l) or buffer were perforrned daily into the anterior chamber of the eye. After 7 days of treatment, the ipsilateral and contralateral irides were examined for content of SP, TH, and CAT. $N=$ 8. *, differs from control at $p<0.005 ; * *$, difters from control at $p<0.05$. sensory, sympathetic, and parasympathetic innervations of the iris are mediated by endogenous NGF.

Effects of AF64A and NGF. The previous observations suggested that the cholinergic ciliary innervation of the iris is responsive to NGF, but quantitatively to a lesser extent than the sensory and sympathetic innervations. To determine whether damaged and/or regenerating ciliary fibers are more responsive to the factor, the cholinergic innervation of the iris was damaged by injection into the anterior chamber of the putative, cholinergic neurotoxin AF64A (Mantione et al., 1981).

Unilateral injections of AF64A or vehicle were performed and repeated the following day. Ipsilateral injections of NGF or vehicle were given simultaneously but were continued daily for 10 days, and the ipsilateral and contralateral irides were examined for CAT and TH activities. The contralateral irides did not differ in any of the groups with respect to $\mathrm{TH}$ or CAT activities, excluding systemic effects of either AF64A or NGF. Administration of AF64A resulted in a $48 \%$ reduction in iris CAT activity, indicating damage to the cholinergic innervation (Fig. 5). However, treatment with AF64A also resulted in a smaller but significant $29 \%$ decrease in iris $\mathrm{TH}$ activity (Fig. 5) and a 20\% decrease in SP (data not shown), indicating a lack of complete spccificity of the toxin. Administration of NGF alone increased CAT activity by $29 \%$. However, treatment with both AF64A and NGF paradoxically elevated CAT activity by an even greater $49 \%$.

Effects of AF64A after sympathetic and sensory denervation. To determine whether damaged cholinergic nerves respond to endog enous NGF after sympathetic and sensory ganglionectomies, AF64A or vehicle was injected twice over 2 days immediately after TG and SCG ablation or sham operations. Ten days later the irides were examined for content of CAT (Fig. 6). Administration of AF64A after sham operation resulted in a $59 \%$ decrease in iris CAT. TG and SCG ablation alone increased CAT activity by $33 \%$. However, TG and SCG ablation combined with AF64A treatment resulted in significantly larger $52 \%$ increase in $\mathrm{CAl}$ activity, suggesting that AF64A potentiates the effects of NGF (Fig. 6).

\section{Discussion}

Target organ regulation of neuronal development and function is a generalized phenomenon found throughout the peripheral (Hamburger, 1934; Hendry and Iversen, 1973; Landmesser and Pilar, 1974) and central (Cowan, 1970; Bjorklund ct al., 1975) nervous systems; conversely, normal target maturation is dependent upon the integrity of its innervation. Less clearly understood are interactions which occur among neuronal populations which innervate the same target. In the present study we have examined interactions among the sensory, sympathetic, and parasympathetic innervations of the iris. Our observations suggest that these three neuronal populations reciprocally modulate each other by altering availability of NGF produced by the target.

TABLE III

Fffects of anti-NGF administration

Unilateral superior cervical ganglionectomy or trigeminal ganglionectomy was performed, and ipsilateral injections of anti-NGF (75 units in $10 \mu l$ ) or control serum were performed daily into the anterior chamber of the eye. After 10 days of treatment, the irides were examined for content of SP (mean picograms per iris \pm SEM), TH (mean picomole product per iris per hour \pm SEM), and CAT (mean nanomole product per iris per hour \pm SEM).

\begin{tabular}{|c|c|c|c|c|c|c|c|}
\hline & $\begin{array}{l}\text { Uninjected } \\
\text { Control }\end{array}$ & $\begin{array}{l}\text { Control } \\
\text { Serum }\end{array}$ & Anti-NGF & $\begin{array}{c}\text { Control } \\
\text { Serum } \\
+ \\
\text { SCG Ablation }\end{array}$ & $\begin{array}{c}\text { Anti-NGF } \\
+ \\
\text { SCG Ablation }\end{array}$ & $\begin{array}{c}\text { Control Serum } \\
+ \\
\text { TG Ablation }\end{array}$ & $\begin{array}{c}\text { Anti-NGF } \\
+ \\
\text { TG Ablation }\end{array}$ \\
\hline $\mathrm{SP}$ & $62 \pm 4$ & $64 \pm 5$ & $42 \pm 3^{2}$ & $105 \pm 9^{2}$ & $50 \pm 3^{a}$ & & \\
\hline CAT & $79 \pm 6$ & $82 \pm 7$ & $77 \pm 8$ & $99 \pm 6^{c}$ & $79 \pm 6$ & $106 \pm 7^{a}$ & $78 \pm 6$ \\
\hline
\end{tabular}

\footnotetext{
${ }^{a}$ Differs from control serum group at $p<0.025$

${ }^{b}$ Differs from control serum group at $p<0.01$.

${ }^{\circ}$ Differs from control serum group at $p<0.05$.
} 


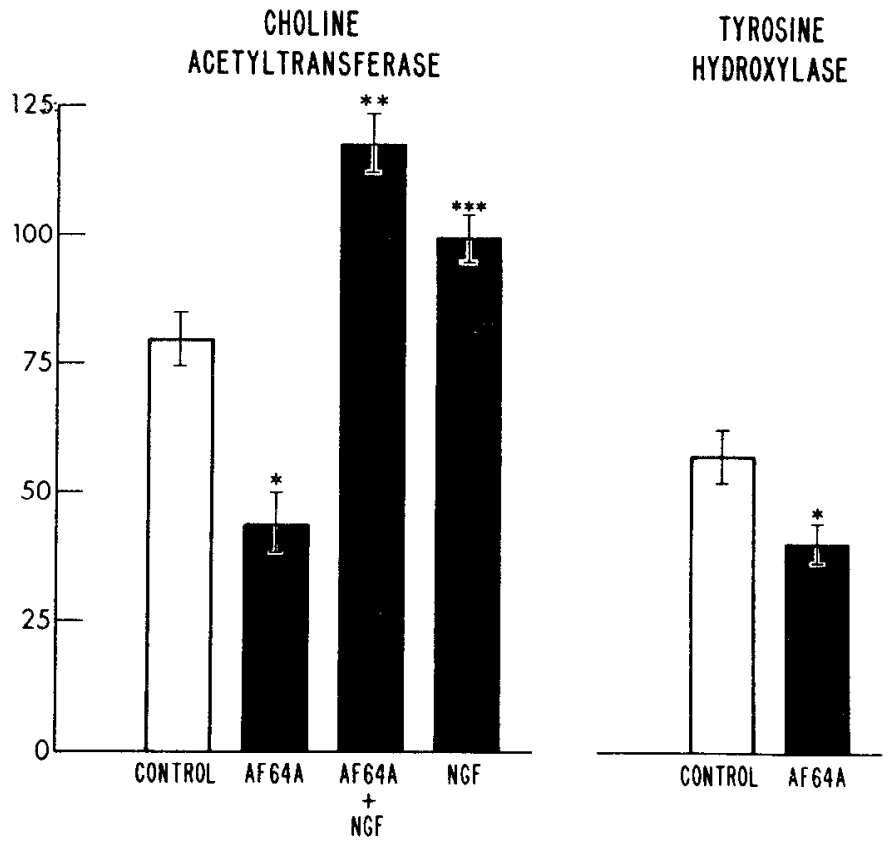

Figure 5. Effects of combined administration of AF64A and NGF into the anterior chamber of the eye. Unilateral injections of AF64A (10 nmol in $10 \mu \mathrm{l})$ or vehicle worc porformcd daily for 2 days. At the same time, ipsilatera injections of NGF ( $4 \mu \mathrm{g}$ in $10 \mu \mathrm{l}$ ) or vehicle were begun and continued daily for 10 days. The ipsilateral and contralateral irides were then examined for content of CAT, TH, and SP. $N=7$. * differs from all other groups at $\rho<$ $0.01 ; * *$, differs from control at $\rho<0.02$ and from NGF-only group at $\rho<$ $0.05 ; * * *$, differs from control at $p<0.05$

Previously we reported that surgical or chemical sympathectomy of the iris increased SP in co-innervating trigeminal tibers, suggesting that sympathetic terminals modulate the sensory peptidergic innervation. In the present study we sought to determine whether the sensory and parasympathetic innervations exert reciprocal effects on sympathetic fibers or on each other. SP was used in this study as an index of the iris sensory innervation, since the peptide is exclusively localized in the iris to trigeminal fibers (Cuello et al., 1978; Butler et al., 1980; Tervo et al., 1982; Kessler et al., 1983a, b). Ablation of the SCG reduced iris $\mathrm{TH}$ to background levels (Table I), suggesting localization exclusively within iris sympathetic fibers; consequently, TH was used as an index of the sympathetic innervation. Finally, ablation of the CG reduced iris CAT activity to minimal levels (Table I); CAT activity was therefore used as a measure of the ciliary innervation.

Trigeminal ablation increased iris TH and CAT activities, indicating that sensory nerves modulate the sympathetic and parasympathetic innervations. Conversely, sympathectomy increased SP and CAT activity, suggesting that sympathetic fibers influence the sensory and parasympathetic iris innervations. Finally, ciliary ganglionectomy increased iris SP and TH activity, indicating that parasympathetic nerves influence the sensory and sympathetic innervations. These reciprocal interactions between the trigeminal, superior cervical, and ciliary fibers clearly indicate that all three nerves interact in establishing the innervation of the iris.

Since NGF apparently mediated the sympathectomy-induced increase in iris SP (Kessler et al., 1983b), we examined the effects of administration of the trophic protein into the anterior chamber. NGF treatment increased CAT and TH activities, as well as SP, indicating that all three iris innervations are responsive to the factor; these observations provide the first evidence that ciliary neurons in vivo are responsive to NGF. Moreover, injection of anti-NGF into the anterior chamber prevented the increases in TH and CAT after trigeminal ablation, as well as the increases in SP and CAT after sympathectomy, suggesting that NGF mediated the rises. Conse- quently, destruction of one source of iris innervation apparently increased availability of NGF to the remaining nerve fibers.

The increased availability of NGF after denervation (Ebendal et al., 1980) may have resulted from decreased competition for existing supplies of the factor, increased synthesis of NGF by the target, or both. Korsching and Thoenen (1983) found detectable NGF in the iris, indicating that the factor is normally present in the innervated iris. Moreover, Shelton and Reichardt (1984) found messenger RNA encoding for NGF (NGF-mRNA) in normally innervated irides, suggesting a normal base line synthesis of the factor. Finally, in this study injection of anti-NGF into the anterior chamber of control animals decreased iris SP and TH activity, suggesting that endogenous NGF normally maintains elevated transmitter levels. Consequently, removal of one source of iris innervation normally competing for the factor should increase availability of NGF to remaining nerve fibers. However, Shelton and Reichardt (1984) also found that denervation of the iris increased levels of NGF-mRNA, suggesting that synthesis of the factor increased after denervation. It seems likely, therefore, that sympathetic, sensory, and parasympathetic fibers exert reciprocal regulatory influences, both by competing for target-derived growth factor and by suppressing target production of the factor. It should be cmphasizcd, however, that factors other than NGF may also be involved; such factors have already been described for both ciliary and sensory neurons. (For review, see Berg, 1984)

The role of NGF in ciliary neuron development and function has been unclear. Max et al. (1978) found that rat and chick ciliary

\section{CHOLINE ACETYLTRANSFERASE}

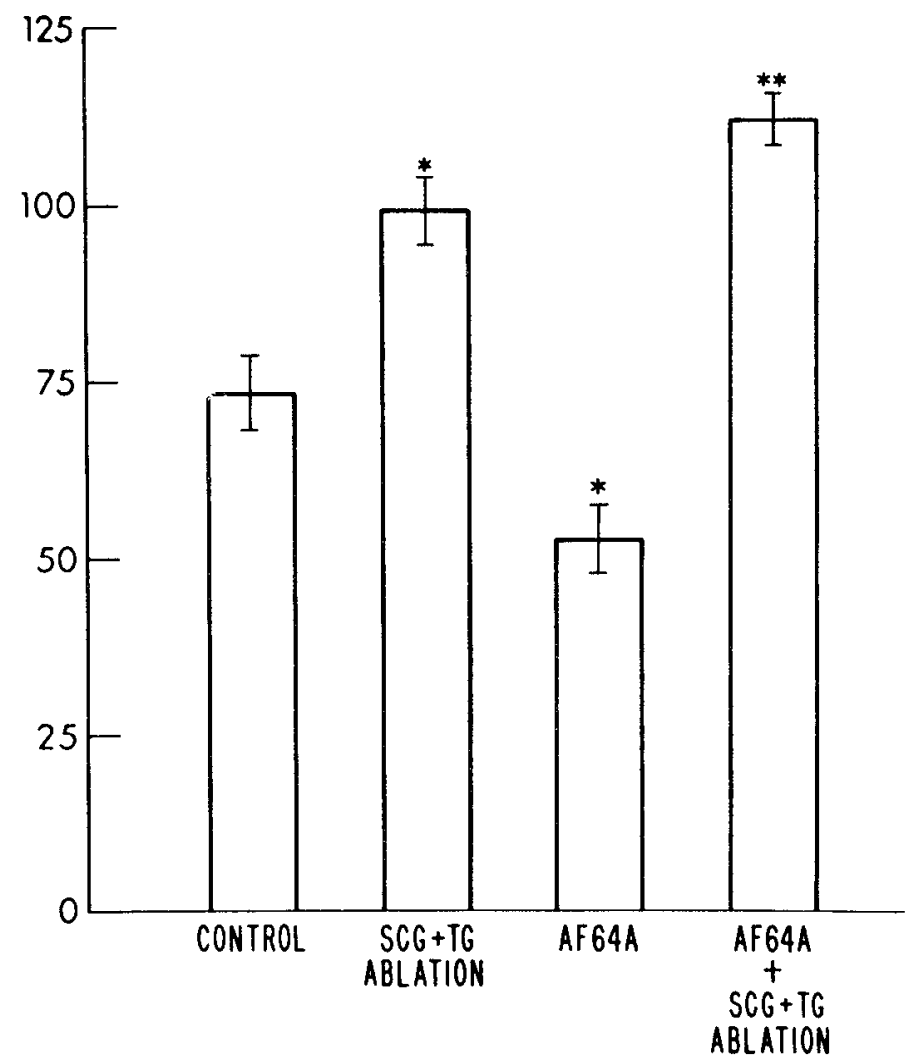

rigure 6. [ffects of combined sympathetic and sensory ganglion ablations and AF64A administration into the anterior chamber. Unilateral SCG and TG ablations or sham operations were performed. Ipsilateral injections of AF64A ( $10 \mathrm{nmol}$ in $10 \mu \mathrm{l}$ ) or vehicle were then performed daily for 2 days. Ten days later the ipsilateral and contralateral irides were examined for content of CAT activity. $N=6$. * differs from control at $p<0.02 ; * *$, differs from control at $p<0.02$ and from SCG and TG ablation at $p<0.05$. 
neurons exhibit specific uptake and retrograde transport of NGF, suggesting a possible role for the factor in ciliary function. Rohrer and Barde (1982), however, reported that chick ciliary neurons have no NGF receptors, although, apparently, non-neuronal cells in the embryonic ganglion transiently express the receptor (Rohrer and Sommer, 1983). Furthermore, Pearson et al. (1983) found no effect of anti-NGF exposure during embryogenesis on ciliary neuron numbers or morphology, indicating that ciliary neurons do not require NGF for survival. Nevertheless, Collins (1984) reported that cultured chick ciliary neurons extend neurites in response to NGF treatment, suggesting that the $\mathrm{CG}$ is responsive to the factor. In the present study, the increase in iris CAT activity after NGF administration into the anterior chamber indicates that parasympathetic fibers can take up the trophic material, and that ciliary neurons possess NGF receptors, the activation of which increases CAT activity. Does endogenous NGF normally regulate the parasympathetic innervation in vivo? The lack of effect on CAT activity of anti-NGF administration into normal eyes, in contradistinction to the decreases in SP and $\mathrm{TH}$, suggests that the factor may not play a significant role in the maintenance of the adult pattern of innervations, although it is possible that a higher dose of anti-NGF might influence CAT activity. However, anti-NGF prevented the increase in CAT activity after damage to the sensory or sympathetic innervations, suggesting that NGF may play a critical role in establishing the pattern of iris innervation. Elaboration of NGF by the developing iris presumably stimulates all three sources of innervation until down regulation of NGF synthesis in response to innervation, and uptake and removal of the factor, by arriving nerve fibers, decrease NGF levels. Moreover, damaged and/or regenerating ciliary fibers may be more responsive to NGF; the cholinergic toxin AF64A and NGF increased iris CAT activity more than NGF alone, suggesting that the toxin enhanced the effects of the trophic protein. Since AF64A also partially damaged the sensory and sympathetic innervations (Fig. $5)$, the augmented increase in CAT activity may have partially reflected elevated endogenous NGF in response to denervation. However, AF64A also enhanced the effects of NGF after prior surgical ablation of the sensory and sympathetic nerves (Fig. 6), suggesting a direct interaction with ciliary fibers. It is of course possible that AF64A affected the iris, which in turn stimulated ciliary CAT activity, and the lack of complete specificity of the drug limits the conclusions which may be drawn. Regardless, it is clear that NGF prevented at least some of the toxic effects of AF64A on cholinergic fibers. This interaction is similar in many respects to the potentiation of NGF effects on noradrenergic neurons after 6-hydroxydopamine treatment (Bjerre et al., 1973; Aloe et al., 1975).

The stimulatory effects of NGF on SP levels and on TH and CAT activities could reflect an increase in the number of nerve fibers (i.e., sprouting), an increase in transmitter trait per fiber, or both. Yodlowski et al. (1984) found an increase in the number of SP-containing iris sensory fibers after sympathectomy and apparent ingrowth of SP containing sensory fibers into sweat glands after sympathetic denervation. These observations suggest that at least some of the effects of NGF reflect sprouting of the iris sensory innervation. Moreover, trigeminal axotomy resulted in increased high affinity uptake of noradrenaline in the iris (unpublished observation), suggesting an incrcase in the density of innervation and, therefore, sprouting of sympathetic fibers. In either case, our observations suggest that sensory, sympathetic, and parasympathetic fibers compete for NGF elaborated by the target iris and thereby exert reciprocal regulatory influences. More generally, targets may regulate innervating fibers derived from different neuronal populations by elaborating a single molecular species. Conversely, nerve terminals derived from functionally dissimilar populations may interact indirectly by regulating production of, and competing for, the same trophic substance elaborated by a mutual target.

\section{References}

Aloe, L., E. Magnaini, and R. Levi-Montalcini (1975) Light and electron microscopic studies on the excessive growth of sympathetic ganglia in rats injected daily from birth with 6OHDA and NGF. Arch. Ital. Biol. 113. 326-353.

Angeletti, P. U. (1971) Chemical sympathectomy in newborn animals. Neuropharmacology 10: 55-59.

Angeletti, P. U., and R. Levi-Montalcini (1970) Sympathetic nerve cell destruction in newborn mammals by 6 -hydroxydopamine. Proc. Natl. Acad. Sci. U. S. A. $65: 114-121$.

Berg, Darwin K. (1984) New neuronal growth factors. Annu. Rev. Neurosci. 7: $149-170$.

Bjerre, B., A. Bjorklund, and W. Mobley (1973) A stimulatory effect by nerve factor on the regrowth of adrenergic nerve fibers in the mouse peripheral tissues after chemical sympathectomy with 6-hydroxydopamine. Z. Zellforsch. 146: 15-43.

Bjorklund, A., B. Johansson, V. Stenevi, and N. -A. Svengaard (1975) Reestablishment of functional connections, by regenerating central adrenergic and cholinergic axons. Nature 253: 446-448.

Butler, J. M., D. Powell, and W. G. Unger (1980) Substance P levels in normal and sensorily denervated rabbit eyes. Exp. Eye Res. 30: 311-313.

Clark, D. W. J., R. Laverty, and E. L. Phelan (1972) Longlasting peripheral and central effects of 6-hydroxydopamine in rats. Br. J. Pharmacol. 44: 233-243.

Collins, F. (1984) An effect of nerve growth factor on the parasympathetic ciliary ganglion. J. Neurosci. 4: 1281-1288.

Cowan, W. M. (1970) Anterograde and retrograde transneuronal degeneration in the central and peripheral nervous system. In Contemporary Research Methods in Neuroanatomy, W. J. H. Nauta and S. O. E. Ebbesson, eds., pp. 217-251, Springer-Verlag, New York.

Cuello, A. C., M. Delfiacco, and G. Paxinos (1978) The central and peripheral ends of the substance $P$-containing sensory neurones in the rat trigeminal system. Brain Res. 152: 499-509.

Ebendal, T., L. Olson, A. Seiger, and K. O. Hediund (1980) Nerve growth factors in the iris. Nature 288: 25-28.

Fenton, E. L. (1970) Tissue culture assay of nerve growth factor and of the specific antiserum. Exp. Cell Res. 59: 383-392.

Finch, L., G. Haeusler, and H. Thoenen (1973) A comparison of the effects of chemical sympathectomy by 6 -hydroxydopamine in newborn and adult rats. Br. J. Pharmacol. 47: 249-260.

Fonnum, F. (1969) Radiochemical microassays for the determination of choline acetyltransferase and acetylcholinesterase activities. Biochem. J. 115: 465-472.

Gamse, R., P. Holzer, and F. Lembeck (1980) Decrease of substance P in primary afferent neurones and impairment of neurogenic plasma extravasalion by capsaicin. Br. J. Phamacol. 68: 207-213.

Hamburger, V. (1934) The effects of wing bud extirpation on the development of the central nervous system in chick embryos. J. Exp. Zool. 68: 448494.

Hendry, I. A., and L. L. Iversen (1973) Reduction in the concentration of nerve growth factor in mice after sialectomy and castration. Nature 243 . 500-504.

Hendry, I. A., and $H$. Thoenen (1974) Changes of enzyme pattern in the sympathetic nervous system of adult mice after submaxillary gland removal: Response to exogenous nerve growth factor. J. Neurochem. 22: 999-1004

Jaim-Etcheverry, G., and L. M. Zieher (1971) Permanent depletion of peripheral norepinephrine in rats treated at birth with 6-hydroxydopamine. Eur. J. Pharmacol. 13: 272-276.

Jansco, G., E. Kiraly, and A. Jansco-Gabor (1977) Pharmacologically induced selective degeneration of chemosensitive sensory neurones. Nature 270 : 741-743.

Kessler, J. A., and I. B. Black (1979) The role of axonal transport in the regulation of enzyme activity in sympathetic ganglia of adult rats. Brain Res. 171: 415-424.

Kessler, J. A., and I. B. Black (1980a) Nerve growth factor stimulates the development of substance $P$ in sensory ganglia. Proc. Natl. Acad. Sci. U. S. A. 77: 649-652.

Kessler, J. A., and I. B. Black (1980b) The effects of nerve growth factor (NGF) and antiserum to NGF on the development of embryonic sympathetic neurons in vivo. Brain Res. 189: 157-168.

Kessler, J. A., and I. B. Black (1982) Regulation of substance $P$ in adult rat sympathetic ganglia. Brain Res. 234: 182-187.

Kessler, J. A., W. O. Bell, and I. B. Black (1983a) Substance P levels differ in sympathetic target organ terminals and ganglion perikarya. Brain Res. 258: $144-146$

Kessler, J. A., W. O. Bell, and I. B. Black (1983b) interactions between the sympathetic and sensory innervation of the iris. J. Neurosci. 3: 13011307.

Korsching, S., and $H$. Thoenen (1983) Nerve growth factor in sympathetic 
ganglia and corresponding target organs of the rat: Correlation with density of sympathetic innervation. Proc. Natl. Acad. Sci. U. S. A. 80: 3513-3516.

Landmesser, L., and G. Pilar (1974) Synapse formation during embryogenesis of ganglion cells lacking a periphery. J. Physiol. (Lond.) 241: 715736.

Levitt, M., S. Spector, A. Sjoerdsma, and S. Udenfriend (1965) Elucidation of the rate-limiting step in norepinephrine biosynthesis in the perfused guinea pig heart. J. Pharmacol. Exp. Ther. 148: 1-8.

Mantione, C., A. Fisher, and I. Hanin (1981) The AF64A-treated mouse: Possible model for central cholinergic hypofunction. Science 213: 579580

Max, S. R., M. Schnub, M. Dumas, and H. Thoenen (1978) Retrograde axonal transport of nerve growth factor in the ciliary ganglion of the chick and the rat. Brain Res. 159: 411-415.

Miller, A., M. Costa, J. B. Furness, and J. W. Chubb (1981) Substance P immunoreactive sensory nerves supply the rat iris and cornea. Neurosci. Lett. 23: 243-249.

Mobley, W. C., A. Schenker, and E. M. Shooter (1976) Characterization and isolation of proteolytically modified nerve growth factor. Biochemistry 15: $5543-5551$

Nagy, J. I. (1982) Capsaicin's action on the nervous system. Trends Neurosci. 5: $362-366$

Nagy, J. I., S. P. Hunt, L. L. Iversen, and P. C. Emson (1981) Biochemical and anatomical observations on the degeneration of peptide-containing primary afferent neurons after neonatal capsaicin. Neuroscience 6: 19231934.

Olson, L., and T. Malmfors (1970) Growth characteristics of adrenergic nerves in the adult rat. Acta Physiol. Scand. Suppl. 348: 1-112.
Otten, N. U., M. Goedert, N. Mayer, and F. Lembeck (1980) Requirement of nerve growth factor for development of substance P-containing sensory neurons. Nature 287: 158-159.

Pearson, J., E. Johnson, and L. Brandeis (1983) Effects of antibiotics to nerve growth factor on intrauterine development of derivatives of cranial neural crest and placode in the guinea pig. Dev. Biol. 96: 32-36.

Prestige, M. C. (1967) The control of cell number in the lumbar spinal ganglia during the development of Xenopus laeuis tadpoles. J. Embryol. Exp. Morphol. 17: 453-471.

Rohrer, $H_{\text {. }}$ and $Y$. A. Rarde (1982) Presence and disappearance of nerve growth factor receptors on sensory neurons in culture. Dev. Biol. 89: 309315.

Rohrer, H., and I. Sommer (1983) Simultaneous expression of neuronal and glial properties by chick ciliary ganglion cells during development. J. Neurosci. 3: 1683-1693.

Shellon, D. L., and L. F. Reichardt (1984) Control of expression of the beta nerve growth factor gene in sympathetic effector organs. Soc. Neurosci. Abstr. 10: 369 .

Tervo, K., T. Tervo, L. Eranko, O. Eranko, S. Valtonen, and A. C. Cuello (1982) Effect of sensory and sympathetic denervation on Substance P immunoreactivity in nerve fibers of the rabbit eye. Exp. Eye Res. 34: 5360

White, H. L. and J. C. Wu (1973) Choline and carnitine acetyltransferases. Biochemistry 12: 841-846.

Yodlowski, M. L., J. R. Fredieu, and S. C. Landis (1984) Neonatal 6hydroxydopamine treatment eliminates cholinergic sympathetic innervation and induces sensory sprouting in rat sweat glands. J. Neurosci. 4: 15351548. 